\title{
Relationships Between Serum Hormone Levels and Semen Quality Among Men From an Infertility Clinic
}

\author{
JOHN D. MEEKER, $*$ LINDA GODFREY-BAILEY $\uparrow \uparrow$ AND RUSS HAUSER $\dagger$ \\ From the *Department of Environmental Health Sciences, University of Michigan School of Public Health, \\ Ann Arbor, Michigan; the †Department of Environmental Health, Harvard School of Public Health, \\ Boston, Massachusetts; and the Vincent Memorial Obstetrics and Gynecology Service, Andrology Laboratory and \\ MGH Fertility Center, Massachusetts General Hospital, Boston, Massachusetts.
}

\begin{abstract}
Participation rates in epidemiologic studies on semen quality are generally very low, raising concerns as to the potential for selection bias. Since hormones both initiate and maintain spermatogenesis, they may serve as surrogates of semen quality in epidemiologic studies. For this reason, in the present study, we explored the influence and predictive ability of reproductive and thyroid hormones on semen quality among men who were partners in an infertile couple. Between 1999 and 2003, 388 men were recruited from Massachusetts General Hospital Andrology Laboratory for clinical evaluation of fertility status. Fresh semen samples were assessed for quality (concentration, motility and morphology) and the serum levels of hormones, including follicle-stimulating hormone (FSH), luteinizing hormone (LH), inhibin $B$, sex hormonebinding globulin (SHBG), testosterone, free androgen index, free $\mathrm{T}_{4}$, total $T_{3}$, and thyroid-stimulating hormone (TSH), were measured. Multiple logistic regression revealed increased odds for belowreference sperm concentration and morphology in men with increased FSH, and decreased odds for below-reference sperm concentration and motility in men with increased inhibin $\mathrm{B}$. When
\end{abstract}

FSH and inhibin B were divided into quintiles, the relationships with sperm concentration showed evidence of a threshold value. However, the ability of specific FSH (10 IU/L) and/or inhibin B $(80 \mathrm{pg} / \mathrm{mL})$ cutoff values to predict semen quality was lower than in previous reports. In multiple linear regression analysis, FSH and $\mathrm{LH}$ were inversely associated with sperm concentration, motility, and morphology. Inhibin $B$ and free $T_{4}$ were positively associated with sperm concentration, while there was a suggestive positive association between testosterone and sperm motility. In conclusion, we have found that $F S H, L H$, inhibin $B$, testosterone and free $T_{4}$ levels are associated with human semen parameters. Additional consideration should be given to the utility of serum hormone levels as a surrogate for semen quality in epidemiologic studies in which the collection of semen is difficult due to logistical and/or volunteer rate constraints.

Key words: Epidemiology, human, male, reproduction, sperm, thyroid.

J Androl 2007;28:397-406
$\mathrm{H}$ ormones play a vital role in initiating and maintaining male reproductive function, yet it is not well understood how variability in the levels of some hormones impact semen quality. In addition, the utility of hormones as predictors of semen quality in epidemiologic studies is of particular interest, as participation rates in epidemiologic studies of semen quality are generally very low, which raises the concern about the potential for selection bias. Previous studies have reported that circulating levels of specific reproductive hormones in men are associated with semen quality parameters (Sina et al, 1975; Subhan et al, 1995; Jensen et al, 1997; Mahmoud et al, 1998). In particular, inhibin B and FSH are thought to be markers of spermatogenesis

Correspondence to: John Meeker, Department of Environmental Health Sciences, University of Michigan School of Public Health, M6226 SPH II, 109 S. Observatory St, Ann Arbor, MI 48109 (e-mail: meekerj@umich.edu).

Received for publication August 22, 2006; accepted for publication November 20, 2006.

DOI: $10.2164 /$ jandrol.106.001545 and Sertoli cell function, and it has even been suggested that measuring the two hormones in serum could serve as a substitute for measuring semen quality or fecundability in epidemiologic studies (Jensen et al, 1997; Pierik et al, 1998, 2003; Uhler et al, 2003; Mabeck et al, 2005). However, the potential relationship between other reproductive hormones, including thyroid hormones, and semen quality has not been studied extensively.

A study of 349 Danish men who had not previously attempted pregnancy found that serum inhibin B was positively correlated with sperm concentration, while FSH was negatively correlated with sperm concentration (Jensen et al, 1997). That study also reported that the predictive power (positive predictive value) of detecting sperm counts below $20 \times 10^{6} / \mathrm{mL}$ in men with inhibin B levels below $80 \mathrm{pg} / \mathrm{mL}$ and with FSH above $10 \mathrm{IU} / \mathrm{L}$ was $100 \%$ (the positive predictive value for individual hormones was $80.0 \%$ and $85.7 \%$ for inhibin B and FSH, respectively). Similarly, in a small Belgian study of 47 subfertile men, Mahmoud and coworkers (1998) have reported higher inhibin B and 
lower FSH levels in men with sperm concentrations greater than or equal to $20 \times 10^{6} / \mathrm{mL}$. They also reported good ability to predict sperm concentrations greater than or less than $20 \times 10^{6} / \mathrm{mL}$ with the same cutoff levels for serum inhibin B (sensitivity $=0.42$; positive predictive value $=100 \%$ ) and FSH (sensitivity $=0.74$; positive predictive value $=96.3 \%$ ). In the US, a prospective clinical study of 145 normal couples of reproductive age with proven fertility found significant positive correlations between inhibin $\mathrm{B}$ and sperm concentration, motility and morphology, and significant negative correlations between FSH and sperm concentration and morphology (Uhler et al, 2003). They reported that 17 of the 22 men in the study with sperm concentrations of less than $20 \times 10^{6} / \mathrm{mL}$ had inhibin B levels of less than $80 \mathrm{pg} / \mathrm{mL}$ and FSH levels of more than $10 \mathrm{IU} / \mathrm{L}$ (sensitivity $=0.77$ ).

Aside from the Danish study (Jensen et al, 1997), previous studies on the relationship between hormone levels and semen quality have been largely conducted on either fertile (Uhler et al, 2003) or infertile (Sina et al, 1975; Subhan et al, 1995; Mahmoud et al, 1998) men. In the present study, the relationship between hormones and semen quality was explored in a population that included men recruited from an infertility clinic as part of an ongoing study of factors involved in altered male reproductive health. The couples who attended the clinic did so because of male factors, female factors or a combination of both male and female factors, so this population included men with normal fertility, subfertility, and male factor infertility. In addition, the previous studies did not measure other hormones, such as LH, testosterone, and thyroid hormones, or they failed to detect associations between these other hormones and semen quality, possibly as a result of small sample sizes or crude statistical analyses. In the present study, we assessed a wider range of reproductive and thyroid hormones among a larger study population, and utilized several statistical modeling approaches to report the degree of observed associations between hormones and semen quality.

\section{Materials and Methods}

\section{Subject Recruitment}

Between 1999 and 2003, men (18-54 years of age) were recruited from the Vincent Memorial Andrology Laboratory at Massachusetts General Hospital and invited to participate in a study to assess the effects of environmental exposure on male reproductive health. Approximately $65 \%$ of the eligible men agreed to participate. The primary reason cited by nonparticipants was a lack of time. Exclusionary criteria included prior vasectomy or current use of exogenous hormones. A retro- spective review of anonymized clinic records of the nonparticipants, who met the same eligibility criteria as the study subjects, found that there were no differences between the participants and nonparticipants in regards to age or semen parameters (Hauser et al, 2005). Height and weight were measured, and all of the men completed a brief nurseadministered questionnaire at the time of recruitment and provided health information. The Harvard School of Public Health (HSPH), Massachusetts General Hospital (MGH), and University of Michigan Human Subjects Committees approved the study, and all subjects signed an informed consent form.

\section{Collection of Semen Samples}

Semen was collected by masturbation into a sterile plastic specimen cup at the hospital. Subjects were instructed to abstain from ejaculation for at least 48 hours prior to producing the semen sample. The sample was liquefied for at least 20 minutes, but no longer than 1 hour prior to performing a routine semen analysis, which included measurements of volume, $\mathrm{pH}$, sperm concentration, sperm motility, progressive motility, and sperm morphology.

\section{Laboratory Evaluation}

Concentration and motility - Semen samples were analyzed for sperm concentration and motion parameters using a computeraided semen analyzer (CASA, version 10 HTM-IVOS; Hamilton Thorne Research, Beverly, Mass). Setting parameters and the definition of measured sperm motion parameters for CASA were established by the manufacturer. To measure both sperm concentration and motility, $5 \mu \mathrm{L}$ of semen from each sample was placed into a prewarmed $\left(37^{\circ} \mathrm{C}\right)$ Makler counting chamber (Sefi Medical Instruments, Haifa, Israel). A minimum of 200 sperm cells from at least four different fields was analyzed from each specimen. Motile sperm were defined according to the World Health Organization (WHO) grade as 'a' grade sperm (rapidly progressive with a velocity $\geq 25 \mu \mathrm{m} / \mathrm{s}$ at $37^{\circ} \mathrm{C}$ ) and 'b' grade sperm (slow/sluggish progressive with a velocity $\geq 5 \mu \mathrm{m} / \mathrm{s}$ but $<25 \mu \mathrm{m} / \mathrm{s}$ ) (WHO, 1999). Progressive motile sperm were defined as grade 'a' sperm (WHO, 1999).

Morphology-At least two slides were made for each fresh semen sample. The resulting thin smear was allowed to air dry for 1 hour before staining with the Diff-Quik staining kit (Dade Behring AG, Dudingen, Switzerland). Morphological assessment was performed with a Nikon microscope using an oil immersion $100 \times$ objective (Nikon Company, Tokyo, Japan). A minimum of 200 sperm cells was counted from the 2 slides for each specimen. Strict scoring criteria were used to classify men as having normal or subnormal morphology (Kruger et al, 1988).

Reproductive Hormones - One nonfasting blood sample was drawn between 0900 and 1600 hours on the same day that the semen sample was collected. Blood samples were centrifuged and the sera were stored at $-80^{\circ} \mathrm{C}$ until analysis. Testosterone was measured directly using the Coat-A-Count RIA kit (Diagnostics Products, Los Angeles, CA), which has interassay and intra-assay coefficients of variation (CV) of $12 \%$ and $10 \%$, respectively, with a sensitivity of $4 \mathrm{ng} / \mathrm{dL}(0.139 \mathrm{nmol} / \mathrm{L})$. The free androgen index (FAI) was calculated as the molar ratio of 
total testosterone to sex hormone-binding globulin (SHBG). SHBG was measured using a fully automated system (Immulite; DPC Inc, Los Angeles, Calif), which uses a solid-phase two-site chemiluminescent enzyme immunometric assay and has an interassay $\mathrm{CV}$ of less than $8 \%$. Inhibin $\mathrm{B}$ was measured using a commercially available, double-antibody, enzyme-linked immunosorbent assay (Oxford Bioinnovation, Oxford, United Kingdom) with interassay and intra-assay CVs of $20 \%$ and $8 \%$, respectively, limit of detection (LOD) of $15.6 \mathrm{pg} / \mathrm{mL}$, and a functional sensitivity $(20 \% \mathrm{CV})$ of $50 \mathrm{pg} / \mathrm{mL}$. The serum luteinizing hormone ( $\mathrm{LH})$ and follicle-stimulating hormone (FSH) concentrations were determined by microparticle enzyme immunoassay using an automated Abbott AxSYM system (Abbott Laboratories, Chicago, Ill). The Second International Reference Preparation (WHO 71/223) was used as the reference standard. The assay sensitivities for LH and FSH were 1.2 IU/L and 1.1 IU/L, respectively. The intra-assay CVs for LH and FSH were less than $5 \%$ and less than $3 \%$, respectively, with interassay $\mathrm{CVs}$ for both hormones of less than $9 \%$. The LH:testosterone ratio, which is a measure of Leydig cell function, was calculated by dividing $\mathrm{LH}(\mathrm{IU} / \mathrm{L})$ by testosterone $(\mathrm{nmol} / \mathrm{L})$.

Thyroid Hormones and TSH-Free $\mathrm{T}_{4}$, total $\mathrm{T}_{3}$, and the TSH concentrations were determined in serum samples by microparticle enzyme immunoassay using the AxSYM system. The assay sensitivities for free $\mathrm{T}_{4}$ and total $\mathrm{T}_{3}$ were $0.01 \mathrm{ng} / \mathrm{dL}$ and $0.15 \mathrm{ng} / \mathrm{mL}$, respectively. The interassay $\mathrm{CVs}$ for both hormones were less than 9\%. For TSH, the ultrasensitive hTSH II assay (Abbott Diagnostics) was used with a functional sensitivity of $0.03 \mu \mathrm{IU} / \mathrm{L}$, and interassay $\mathrm{CVs}$ of less than $8 \%$.

\section{Statistical Analysis}

Data analysis was performed using SAS version 8.1 (SAS Institute Inc, Cary, NC). Descriptive statistics on subject demographics were calculated, along with the distributions of hormone levels and semen parameters. Hormone levels and semen parameters were stratified by demographic categories to investigate the potential for confounding. Spearman correlation coefficients were used to determine correlations among hormones, among semen parameters, and between semen parameters and hormone levels.

Statistical analyses were performed using semen parameters as both a continuous measure and dichotomized using WHO reference values for sperm concentration $\left(<20 \times 10^{6} / \mathrm{mL}\right)$ and motility ( $<50 \%$ motile sperm) (WHO, 1999). The Tygerberg Strict Criteria for morphology were used to determine belowreference morphology ( $<4 \%$ normal morphology) (Kruger et al, 1988). Men with above-reference values for all three semen parameters were used as comparison subjects in multivariate logistic regression. Using logistic regression to explore the shape of the hormone-semen quality relationships, the hormone levels were categorized in tertiles, and then in quintiles. To compare the abilities of serum inhibin $\mathrm{B}$ and FSH to predict sperm concentrations below the WHO reference level in the present study with those reported in previous studies (Jensen et al, 1997; Mahmoud et al, 1998), the sensitivity, specificity, and positive predictive values were calculated using the same criteria (inhibin $\mathrm{B}<80 \mathrm{pg} / \mathrm{mL}$ and FSH $<10$ IU/L).
Multivariate linear regression was used to explore the continuous relationships between hormones and semen parameters. The concentrations of testosterone, inhibin $\mathrm{B}$, free $\mathrm{T}_{4}$, and total $\mathrm{T}_{3}$ closely approximated normality and were used untransformed in statistical models, while the distributions of the FSH, LH, SHBG, FAI, and TSH concentrations were skewed right and transformed to the natural $\log (\mathrm{ln})$ for statistical analyses. Sperm concentration was also ln-transformed, while sperm motility and morphology were modeled untransformed. To improve interpretability, the regression coefficients were back-transformed and expressed as a change in the dependent variable (ie, semen parameters) for an interquartile range (IQR) increase in hormone levels.

Inclusion of covariates was based on statistical and biologic considerations (Hosmer and Lemeshow, 1989). Age and BMI were modeled as a continuous variable, smoking status was dichotomized by current smoker versus never smoked or former smoker, and race/ethnicity was categorized into four groups: White, African-American, Hispanic, and other. Previous exam for infertility (yes or no), prior ability to impregnate a partner (yes or no), and timing of blood sample by season (Winter vs Spring, Summer or Fall) and time of day (0900-1259 hours vs 1300-1600 hours) were considered for inclusion as dichotomous variables in the models.

\section{Results}

Of the 522 men recruited, 56 were excluded owing to self-reported medical risk factors for infertility (eg, varicocele or orchidopexy). An additional 13 subjects were excluded for taking hormone medications. Of the remaining 453 men, 388 provided both semen samples and serum for hormone level measurements (hormone levels were unavailable for 63 men and semen parameters were unavailable from $2 \mathrm{men}$ ), and these men comprised the final study population in the present study. Among the 388 subjects (Table 1), the majority were white $(85 \%)$ and had never smoked $(71 \%)$. The mean (SD) age and BMI were 36 (5.4) years and 28 (4.6), respectively. The distributions of semen parameters and hormone levels are presented in Tables 2 and 3, respectively. Most men in the study had hormone levels within the MGH reference ranges, where applicable.

In the preliminary bivariate analyses, there was a significant positive (linear) association between the ordinal abstinence categories and sperm concentration, and an inverse (linear) association between abstinence and sperm morphology. For the hormones, FSH was positively correlated with $\mathrm{LH}$ and inversely correlated with inhibin B. Age was associated with decreased FAI, free $T_{4}$, and total $T_{3}$, and BMI was associated with decreased inhibin B, testosterone, and SHBG. There was a positive association between BMI and FAI. There were positive associations between the ordinal absti- 
Table 1. Demographic categories by semen parameters*

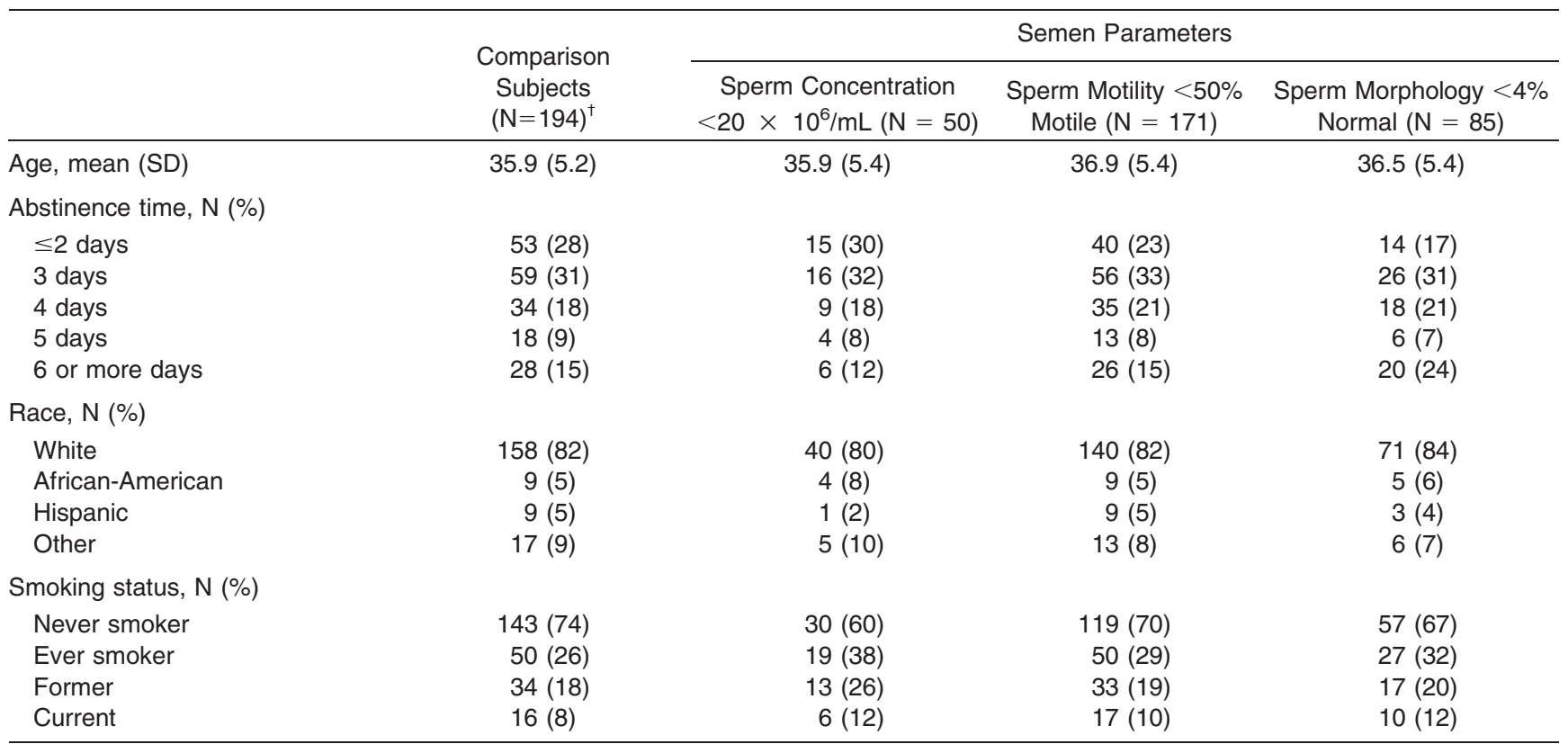

* Information on race missing for 1 man, on smoking for 4 men, and on abstinence time for 4 men.

† Comparison group consists of men with all 3 semen parameters above the reference level.

nence time categories and $\mathrm{LH}$ and $\mathrm{FSH}$, where men with abstinence times of more than 6 days had higher LH and FSH levels than men who abstained for 3 or fewer days. Current smokers had higher total $\mathrm{T}_{3}$ concentrations and lower TSH concentrations than former and non-smokers. Inhibin $\mathrm{B}$ and free $\mathrm{T}_{4}$ levels were lower in men who had blood samples collected in Winter than in men with samples collected in the Spring, Summer or Fall, while blood samples collected in the morning (between 0900 and 1259) showed higher inhibin B and testosterone levels than those collected in the afternoon.

Using multivariate logistic regression analysis, semen parameters (dichotomized based on WHO reference cutoff points) were regressed on hormone tertiles (Table 4). Compared with men in the lowest FSH tertile, men in the highest FSH tertile were nearly five-times more likely to have below-reference $\left(<20 \times 10^{6} / \mathrm{mL}\right)$ sperm concentrations (odds ratio $[\mathrm{OR}]=4.6 ; 95 \%$ confidence intervals [CI] 1.9 to 11.2). In addition, men in the highest FSH tertile were more than 2 times more likely to have below-reference $(<4 \%$ normal) sperm morphology (OR
$=2.3 ; 95 \%$ CI 1.2 to 4.4$)$. The highest LH tertile was also associated with below-reference sperm concentration $(\mathrm{OR}=3.9 ; 95 \%$ CI 1.7 to 9.2$)$, as was the highest LH:testosterone ratio tertile. Conversely, men in the medium and highest inhibin B tertiles were more likely to have above-reference sperm concentrations (high inhibin $\mathrm{B}$ tertile $\mathrm{OR}=0.2 ; 95 \% \mathrm{CI} 0.1$ to 0.5 ) and sperm morphology (high inhibin B tertile OR $=0.6 ; 95 \%$ CI 0.3 to 1.1) compared to the lowest tertile. These associations, some of which did not appear to be monotonic, were also observed when the hormone levels were further divided into quintiles (Figures 1 and 2). The increased odds for below-reference sperm concentration among only the highest FSH quintile and among only the lowest inhibin B quintile may support the notion of a threshold for belownormal sperm concentration in association with these hormones. However, the abilities of inhibin B and FSH to predict below-reference sperm concentration were limited. Of only 26 men with both inhibin B of less than $80 \mathrm{pg} / \mathrm{mL}$ and FSH greater than $10 \mathrm{IU} / \mathrm{L}, 18(69 \%)$ had sperm concentrations less than or equal to $20 \times 10^{6} / \mathrm{mL}$.

Table 2. Distribution of semen parameters for 388 male subjects

\begin{tabular}{lcrrrrrrr}
\hline & & \multicolumn{9}{c}{ Selected Percentiles } \\
\cline { 3 - 8 } \multicolumn{1}{c}{ Parameter } & Mean & 5th & 10th & 25th & 50th & 75th & 90th & 95th \\
\hline Sperm concentration $\left(10^{6} / \mathrm{mL}\right)$ & 113 & 4 & 14 & 35 & 83 & 168 & 236 & 269 \\
Sperm motility $(\%)$ & 50.3 & 7 & 14 & 32 & 54 & 70 & 78 & 83 \\
Sperm morphology (\% normal) & 7.2 & 0 & 1 & 4 & 7 & 10 & 14 & 15 \\
\hline
\end{tabular}


Table 3. Distribution of serum hormone levels for 388 male subjects

\begin{tabular}{|c|c|c|c|c|c|c|c|c|}
\hline \multirow[b]{2}{*}{ Hormone } & \multirow[b]{2}{*}{ Mean } & \multicolumn{7}{|c|}{ Selected Percentiles } \\
\hline & & 5th & 10th & 25th & 50th & 75th & 90th & 95th \\
\hline FSH (IU/L) & 9.18 & 3.65 & 4.33 & 5.59 & 7.28 & 10.0 & 14.7 & 20.9 \\
\hline LH (IU/L) & 11.2 & 4.83 & 5.46 & 7.26 & 9.77 & 13.3 & 17.2 & 20.4 \\
\hline Inhibin B (pg/mL) & 168 & 67.3 & 86.9 & 122 & 164 & 199 & 256 & 283 \\
\hline SHBG $(\mathrm{nmol} / \mathrm{mL})$ & 28.1 & 12.6 & 15.3 & 20.0 & 26.1 & 33.9 & 43.5 & 49.9 \\
\hline Testosterone (ng/dL) & 419 & 221 & 251 & 321 & 406 & 493 & 603 & 655 \\
\hline FAI & 0.57 & 0.31 & 0.35 & 0.42 & 0.52 & 0.68 & 0.84 & 0.94 \\
\hline LH:Testosterone & 0.93 & 0.36 & 0.40 & 0.52 & 0.70 & 0.94 & 1.33 & 1.70 \\
\hline Free $\mathrm{T}_{4}(\mathrm{ng} / \mathrm{dL})$ & 1.25 & 0.93 & 0.97 & 1.06 & 1.20 & 1.40 & 1.56 & 1.72 \\
\hline Total $\mathrm{T}_{3}(\mathrm{ng} / \mathrm{mL})$ & 0.97 & 0.73 & 0.77 & 0.83 & 0.96 & 1.08 & 1.21 & 1.28 \\
\hline $\mathrm{TSH}(\mu \mathrm{IU} / \mathrm{mL})$ & 1.67 & 0.62 & 0.77 & 1.02 & 1.43 & 1.92 & 2.68 & 3.58 \\
\hline
\end{tabular}

The results from the multivariate linear regression analyses, which are similar to the crude linear regression results, are presented in Table 5. Consistent with the logistic regression results, FSH was inversely associated with sperm concentration and morphology. An IQR increase in FSH was associated with a $38 \%(95 \%$ CI $45 \%$ to $29 \%$ ) decline in sperm concentration and a $0.89 \%$ decline in the percentage of sperm with normal morphology. For the median level of sperm with normal morphology (7\%), this represents an approximate $13 \%$ (95\% CI $20 \%$ to $5 \%$ ) decline in sperm morphology for an IQR increase in FSH. LH was also inversely associated with sperm concentration and morphology. An IQR increase in $\mathrm{LH}$ was associated with a $31 \%(95 \%$ CI $41 \%$ to $20 \%$ ) decline in sperm concentration and a $9 \%$ decline $(95 \%$ CI $17 \%$ to $<1 \%$ ) in the median percentage of sperm with normal morphology (7\%). Unlike the logistic regression results, IQR increases in both FSH and $\mathrm{LH}$ were associated with $4.5 \%$ declines in sperm motility. Associations between semen parameters and $\mathrm{LH}$ :testosterone ratios were similar to those of $\mathrm{LH}$, although the inverse association between LH:testosterone and sperm motility appeared slightly stronger.

Higher inhibin $\mathrm{B}$ and free $\mathrm{T}_{4}$ levels were associated with higher sperm concentrations. IQR increases in inhibin $\mathrm{B}$ and free $\mathrm{T}_{4}$ were associated with $29 \%$ (95\% CI $15 \%$ to $39 \%$ ) and $27 \%$ (95\% CI $4 \%$ to $63 \%$ ) increases in sperm concentration, respectively. Finally, there was a suggestive positive association between testosterone and sperm motility. Interestingly, when men were stratified by motility reference level ( $\geq 50 \%$ motile sperm), testosterone was associated with above-reference motility $(P=.007)$ but not with below-reference motility $(P=.70)$.

\section{Discussion}

The present study shows that the serum levels of FSH and LH are inversely associated with sperm concentration, motility, and morphology, while inhibin $\mathrm{B}$ and free $\mathrm{T}_{4}$ are positively associated with sperm concentration. Our findings for inhibin $\mathrm{B}, \mathrm{FSH}$, and sperm concentrations are consistent with those in several previous studies (Sina et al, 1975; Subhan et al, 1995; Jensen et al, 1997; Mahmoud et al, 1998; Pierik et al, 1998; Mabeck et al, 2005), while only two previous studies have reported associations of inhibin B and FSH with sperm motility and morphology. Jensen et al (2004) have reported significant correlations of FSH, LH, and inhibin B with sperm concentration, motility, and morphology among 1558 young Danish men who reported for military service, although the robustness of these associations was not tested further in multivariate models. In another study, Uhler et al (2003) also have reported significant bivariate correlations of FSH and inhibin B with sperm concentration, motility, and morphology among 145 men in couples with no known fertility impairment, although they found no associations between $\mathrm{LH}$ or testosterone and semen quality parameters, and again, the associations were not tested further in multivariate analyses. We were unable to locate any previous studies that report associations between $\mathrm{LH}$ and multiple semen parameters or that support our observation of a suggestive association between testosterone and sperm motility.

FSH, which is a gonadotropin that is produced and secreted by the anterior pituitary, acts on Sertoli cells in the seminiferous tubules to initiate spermatogenesis. Sertoli cells secrete inhibin B, which is a protein hormone. Thus, as found in previous studies, an association between inhibin B and sperm concentration was to be expected, since the regulation of both factors is dependent upon Sertoli cell function. In addition, the inverse associations of FSH with inhibin B and with sperm concentration may be due to the feedback effects exerted by inhibin B on the anterior pituitary to inhibit FSH secretion (Anawalt et al, 1996; Vander et al, 1998). Information on the specific mechanisms underlying the other observations made in the present study is limited, 


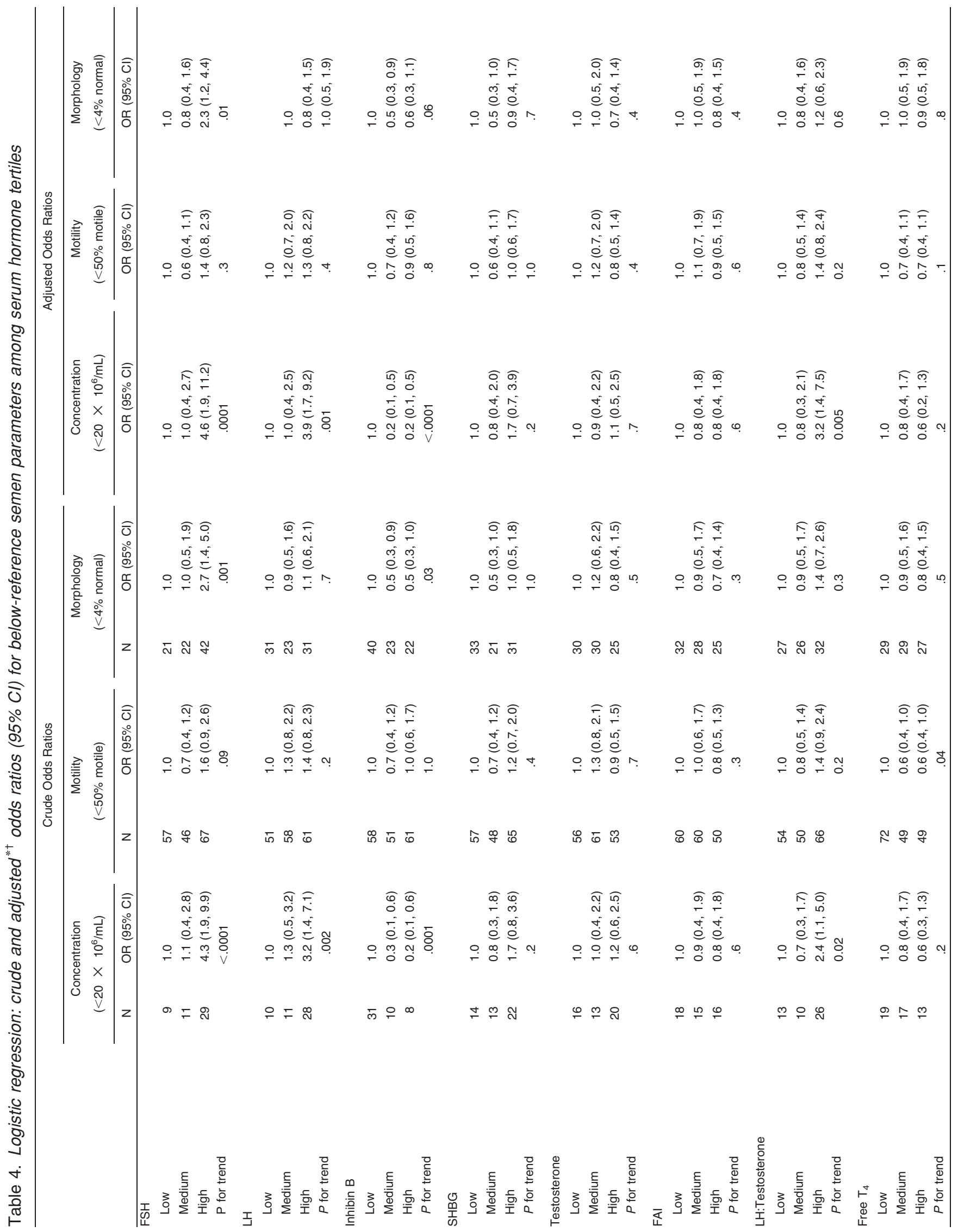




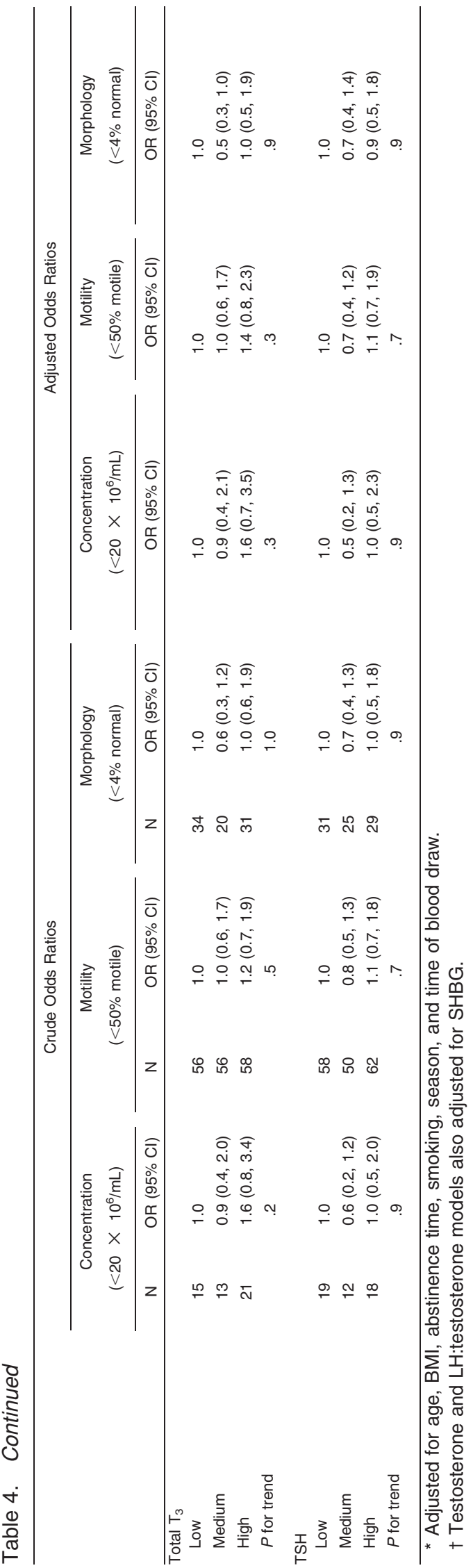

although they may also be related to altered spermatogenesis. Our results suggest that FSH, LH, and inhibin B play roles in sperm development (morphology), whereby increased levels of FSH and $\mathrm{LH}$, but decreased levels of inhibin $\mathrm{B}$, reduce the proportions of normal sperm. Our results also suggest that FSH, LH, and testosterone impact sperm motion (motility). Testosterone facilitates spermatogenesis locally in the Sertoli cells as a paracrine agent, and also serves to provide feedback for GnRH and LH secretion, which suggests that the observed associations with semen parameters involving testosterone (increased sperm motility) and LH (decreased sperm concentration, motility, and morphology) in the present study are related to disruptions and compensatory mechanisms in the hypothalamo-pituitary-gonadal axis.

In the present study, multiple logistic regression revealed an association between free $\mathrm{T}_{4}$ and reduced odds of having sperm motility of less than $50 \%$, while multiple linear regression showed a positive association between free $T_{4}$ and sperm concentration. While infertility is a common clinical manifestation of thyroid hormone deficiency (Nussey and Whitehead, 2001), previous reports of subclinical changes in thyroid hormone levels and semen quality could not be located. Thus, more research is necessary to determine the potential roles of thyroid hormones in semen quality and male fertility.

Consistent with previous studies, we found evidence of a threshold for increased risk of having a sperm concentration of less than $20 \times 10^{6} / \mathrm{mL}$ among men in either the highest serum FSH quintile or the lowest serum inhibin B quintile (Figure 1). However, when using previously published hormone cutoff levels $(>10$ IU/L FSH and $<80 \mathrm{pg} / \mathrm{mL}$ inhibin B), the ability in the present study to predict men with sperm concentrations of less than $20 \times 10^{6} / \mathrm{mL}$ was lower than that described in earlier reports (Jensen et al, 1997; Mahmoud et al, 1998; Uhler et al, 2003). In the present study, an FSH concentration greater than $10 \mathrm{IU} / \mathrm{L}$ was predictive a sperm concentration of less than $20 \times 10^{6} / \mathrm{mL}$ with a sensitivity of 0.55 (specificity $=0.79$; positive predictive value $=28 \%$ ). An inhibin B concentration of less than $80 \mathrm{pg} / \mathrm{mL}$ had a lower sensitivity (0.38) than FSH for predicting below-reference sperm concentration ( specificity $=0.96$; positive predictive value $=56 \%$ ). For 26 of the 388 men with both FSH greater than 10 IU/L and inhibin B less than $80 \mathrm{pg} / \mathrm{mL}$, only 18 had a sperm concentration of less than $20 \times 10^{6} / \mathrm{mL}$ (positive predictive value $=69 \%$ ). Thus, although we found evidence of FSH and inhibin B thresholds for having below-reference sperm concentration, the present study does not fully support using hormone levels to predict semen quality in epidemiology studies based on 


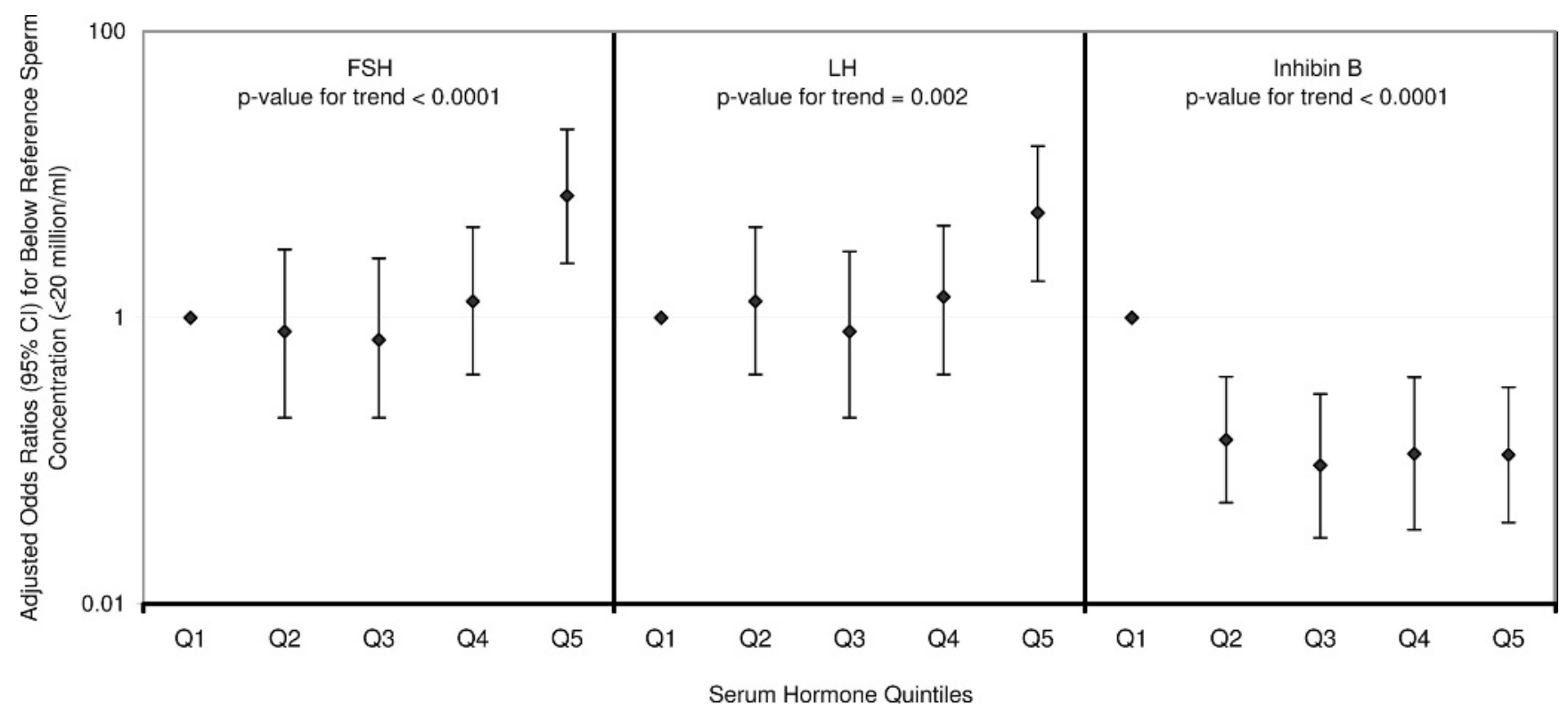

Figure 1. Adjusted odds ratios and $95 \%$ confidence intervals for below-reference sperm concentrations associated with increasing quintiles of $\mathrm{FSH}$, LH, or inhibin B. Data are adjusted for age, BMI, abstinence time, current smoking, season, and time of blood sample. $\mathrm{N}=388$.

these FSH and/or inhibin B cutoff values, as has been proposed previously (Jensen et al, 1997; Uhler et al, 2003). However, we are currently unable to comment on the ability of inhibin B to predict fecundability (Mabeck et al, 2005) or other markers of spermatogenesis (Pierik et al, 1998, 2003).

In the present study, we found associations between hormones and several covariates under consideration as potential confounders (age, BMI, smoking, abstinence time, season, time of day). Of these, several have been reported previously. For example, smoking has been associated with lower TSH levels in the general population (Schlienger et al, 2003). Consistent with our bivariate BMI analysis, a recent study of over 1500 Danish men has shown that increased BMI is associated with reduced levels of inhibin $\mathrm{B}$, testosterone, and SHBG, and with increased FAI (Jensen et al, 2004). In the present study, we observed higher inhibin B and

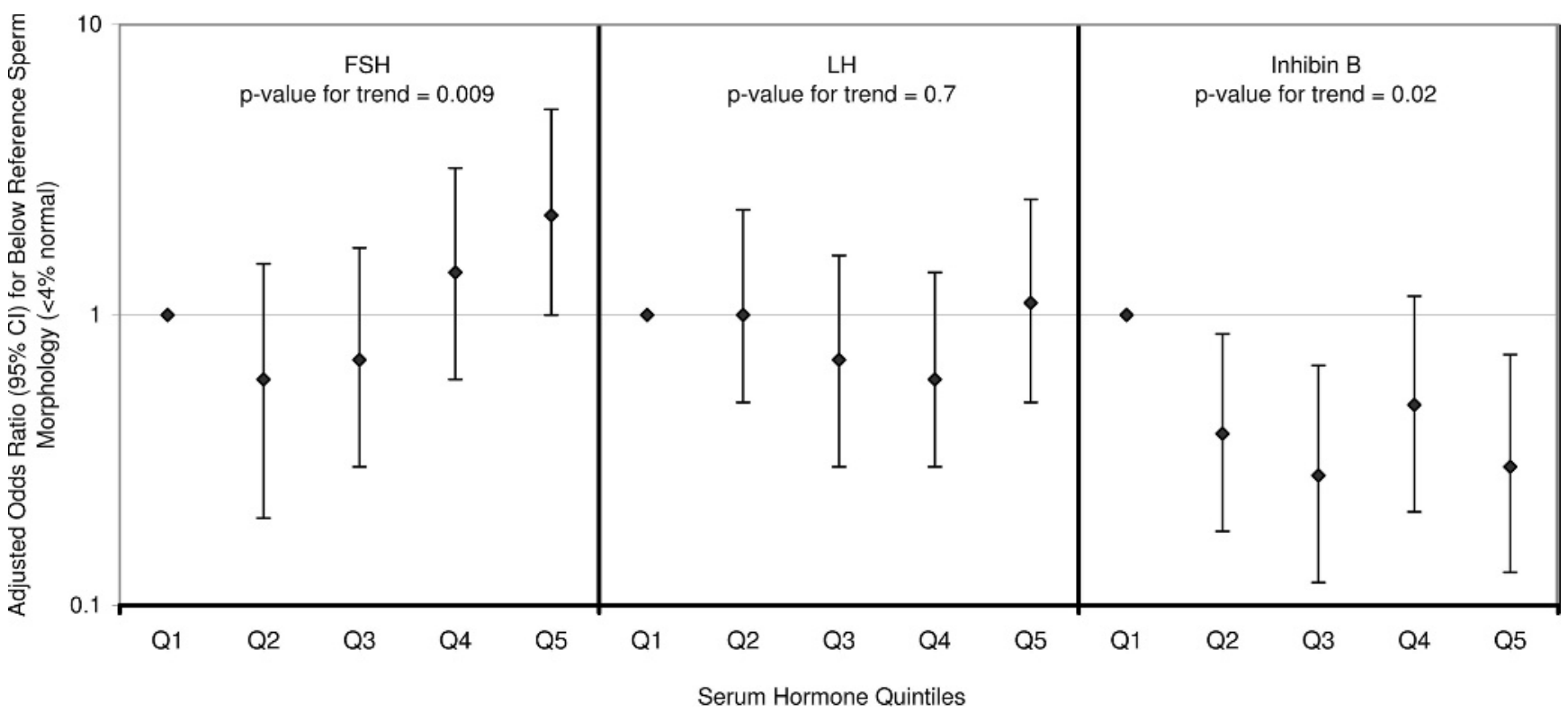

Figure 2. Adjusted odds ratios and $95 \%$ confidence intervals for below-reference sperm morphology associated with increasing quintiles of $\mathrm{FSH}$, LH, or inhibin B. Data are adjusted for age, BMI, abstinence time, current smoking, season, and time of blood sample. $\mathrm{N}=388$. 
Table 5. Adjusted ${ }^{* \dagger}$ linear regression coefficients for change in semen parameters associated with an interquartile range (IQR) increase in serum hormone levels for 388 male subjects

\begin{tabular}{|c|c|c|c|}
\hline & \multicolumn{3}{|c|}{ Adjusted coefficients $(95 \% \mathrm{Cl})^{\ddagger}$} \\
\hline & Concentration $\left(10^{6} / \mathrm{mL}\right)^{\S \|}$ & Motility (\% motile) & Morphology (\% normal) \\
\hline $\mathrm{FSH}^{\S}$ & $0.62(0.55,0.71)^{*}$ & $-4.47(-7.15,-1.80)^{*}$ & $-0.89(-1.40,-0.38)^{*}$ \\
\hline $\mathrm{LH}^{\S}$ & $0.69(0.59,0.80)^{*}$ & $-4.53(-7.69,-1.38)^{*}$ & $-0.61(-1.21,-0.01)^{*}$ \\
\hline InhibinB & $1.29(1.15,1.39)^{*}$ & $2.08(-0.77,4.62)$ & $0.31(-0.15,0.77)$ \\
\hline $\mathrm{SHBG}^{\S}$ & $0.97(0.83,1.13)$ & $-0.32(-3.60,2.97)$ & $-0.36(-0.99,0.26)$ \\
\hline Testosterone $^{\dagger}$ & $0.98(0.83,1.17)$ & $3.61(-0.17,6.88)^{* \star}$ & $-0.34(-1.03,0.34)$ \\
\hline FAl & $1.02(0.90,1.23)$ & $1.97(-1.08,5.02)$ & $-0.12(-0.71,0.46)$ \\
\hline LH:Testosterone ${ }^{\dagger}$ & $0.70(0.60,0.81)^{*}$ & $-6.07(-9.14,-2.99)^{*}$ & $-0.46(-1.05,0.14)$ \\
\hline Free $\mathrm{T}_{4}$ & $1.27(1.04,1.63)^{*}$ & $0.39(-3.01,3.77)$ & $0.14(-0.51,0.79)$ \\
\hline Total $\mathrm{T}_{3}$ & $0.93(0.84,1.10)$ & $-1.10(-4.58,2.40)$ & $-0.36(-1.02,0.31)$ \\
\hline $\mathrm{TSH}^{\S}$ & $0.93(0.83,1.05)$ & $-0.34(-2.92,2.25)$ & $0.09(-0.40,0.58)$ \\
\hline
\end{tabular}

* Adjusted for age, BMI, abstinence time, current smoking, season (winter vs other season), and time (morning vs afternoon) of blood sample.

$\dagger$ All the testosterone and LH:testosterone models are also adjusted for SHBG.

$\ddagger$ In the models in which both independent (FSH, LH, SHBG, and TSH) and dependent (sperm concentration) variables are In-transformed, the results are presented as multiplicative factors for a change in semen parameter associated with an IQR change in hormone level where values less than 1.0 represent multiplicative decreases in semen parameters and values greater than 1.0 represent multiplicative increases in semen parameters. Results from models in which only the semen parameter (sperm concentration) was transformed also represent a multiplicative factor per IQR change in hormone level. For models in which the hormone values are In-transformed but the semen parameters (motility and morphology) are not, the results are presented as changes in semen parameters for an IQR change in hormone level. The results are also presented as a change in semen parameter per IQR change in hormone level when neither the hormone (inhibin B, testosterone, $\mathrm{LH}$ :testosterone ratio, $\mathrm{FAl}$, free $\mathrm{T}_{4}$, and total $\mathrm{T}_{3}$ ) nor the semen parameters (sperm motility and morphology) were transformed.

$\S$ Variable is In-transformed.

II Coefficients represent a multiplicative factor.

$* P<.05$

** $P=.057$

testosterone levels in samples collected in the morning than in those collected in the afternoon, which is consistent with published reports (Veldhuis et al, 1987; Carlsen et al, 1999). In contrast to our observation that inhibin B levels were lower among blood samples collected in Winter, a recent study has reported no seasonal variation in inhibin B levels among healthy men (Andersson et al, 2003). However, our analysis of seasonal inhibin B variation did not simultaneously take into account the time of day of sample collection. For semen quality parameters, our observations of a positive association between abstinence time and sperm concentration and negative association between abstinence time and sperm morphology are consistent with the findings presented in a previous report (Pellestor et al, 1994).

A potential limitation of the present study was the collection of only a single semen sample to assess semen parameters, and the collection of a single blood sample to measure serum hormone levels. However, our analyses were adjusted for several factors associated with interindividual and intraindividual variability in hormone levels and semen quality, and the collection of a single sample is not expected to greatly affect our results. For example, despite the diurnal and pulsatile fluctuations in serum hormone levels, a single blood sample can be used to provide a reliable measure of reproductive hormones over both short and long time periods in population studies (Vermeulen and Verdonck, 1992; Schrader et al, 1993; Andersson et al, 2003). In addition, a requirement for multiple blood samples may limit participation rates in epidemiologic studies (Schrader et al, 1993). Collection of only one semen sample is also not expected to affect our results. Uhler and coworkers (2003) collected multiple semen samples (between 2 and 4) from each subject at time intervals that coincided with their partners' menstrual cycles, but found that the subsequent semen samples did not add value compared to using only the first semen sample collected on the same day as the serum hormone measures.

In conclusion, we show that several hormones are associated with semen quality parameters among a cohort of men from an infertility clinic, which included both infertile men and fertile men who were partners in an infertile relationship. Several of the associations were consistent with previous reports, while others have not been reported elsewhere. Consistent with previous studies, our results showing strong associations between semen quality and FSH and inhibin B and evidence of a threshold support the use of FSH and/or inhibin B as surrogates for semen quality in reproductive epidemio- 
logic studies that are prone to low participation rates for semen sample collection. However, following sensitivity calculations in our population, which included both fertile and infertile men, we found that these hormones have lower predictive abilities than previously reported in other populations. Further investigations of additional male populations are needed for a better understanding of the relationship between hormones and semen quality.

\section{Acknowledgments}

The authors thank Dr Zuying Chen (former Director of the MGH Andrology Laboratory) for semen analysis, literature review, and discussion of the study design, Ms Janna Frelich, HSPH Data Analyst, for dataset management, and Dr Kimberly Pearson for critical reading of the statistical sections.

\section{References}

Anawalt BD, Bebb RA, Matsumoto AM, Groome NP, Illingworth PJ, McNeilly AS, Bremner WJ. Serum inhibin B levels reflect Sertoli cell function in normal men and men with testicular dysfunction. J Clin Endocrinol Metab. 1996;81:3341-3345.

Andersson AM, Carlsen E, Petersen JH, Skakkebaek NE. Variation in levels of serum inhibin $\mathrm{B}$, testosterone, estradiol, luteinizing hormone, follicle-stimulating hormone, and sex hormone-binding globulin in monthly samples from healthy men during a 17-month period: possible effects of seasons. J Clin Endocrinol Metab. 2003;88:932-937.

Carlsen E, Olsson C, Petersen JH, Andersson AM, Skakkebaek NE. Diurnal rhythm in serum levels of inhibin B in normal men: relation to testicular steroids and gonadotropins. J Clin Endocrinol Metab. 1999;84:1664-1669.

Hauser R, Godfrey-Bailey L, Chen Z. Does the potential for selection bias in semen quality studies depend on study design? Experience from a study conducted within an infertility clinic. Hum Reprod. 2005;20:2579-2583.

Hosmer DW Jr, Lemeshow S. Model building strategies and methods for logistic regression. In: Applied Logistic Regression. New York: John Wiley \& Sons; 1989:82-134.

Jensen TK, Andersson AM, Hjollund NH, Scheike T, Kolstad H, Giwercman A, Henriksen TB, Ernst E, Bonde JP, Olsen J, McNeilly A, Groome NP, Skakkebaek NE. Inhibin B as a serum marker of spermatogenesis: correlation to differences in sperm concentration and follicle-stimulating hormone levels. A study of 349 Danish men. J Clin Endocrinol Metab. 1997;82:4059-4063.
Jensen TK, Andersson AM, Jorgensen N, Andersen AG, Carlsen E, Petersen JH, Skakkebaek NE. Body mass index in relation to semen quality and reproductive hormones among 1,558 Danish men. Fertil Steril. 2004;82:863-870.

Kruger TF, Acosta AA, Simmons KF, Swanson RJ, Matta JF, Oehninger $S$. Predictive value of abnormal sperm morphology in in vitro fertilization. Fertil Steril. 1988;49:112-117.

Mabeck LM, Jensen MS, Toft G, Thulstrup M, Andersson M, Jensen TK, Giwercman A, Olsen J, Bonde JP. Fecundability according to male serum inhibin B-a prospective study among first pregnancy planners. Hum Reprod. 2005;20:2909-2915.

Mahmoud AM, Comhaire FH, Depuydt CE. The clinical and biologic significance of serum inhibins in subfertile men. Reprod Toxicol. 1998;12:591-599.

Nussey SS, Whitehead SA. Endocrinology: An Integrative Approach. Oxford, United Kingdom: BIOS Scientific Publishers Ltd; 2001.

Pellestor F, Girardet A, Andreo B. Effect of long abstinence periods on human sperm quality. Int J Fertil Menopausal Stud. 1994;39: 278-282.

Pierik FH, Burdorf A, de Jong FH, Weber RF. Inhibin B: a novel marker of spermatogenesis. Ann Med. 2003;35:12-20.

Pierik FH, Vreeburg JT, Stijnen T, De Jong FH, Weber RF. Serum inhibin B as a marker of spermatogenesis. $J$ Clin Endocrinol Metab. 1998;83:3110-3114.

Schlienger JL, Grunenberger F, Vinzio S, Goichot B. Smoking and the thyroid. Ann Endocrinol (Paris). 2003;64:309-315.

Schrader SM, Turner TW, Breitenstein MJ, Simon SD. Measuring male reproductive hormones for occupational field studies. J Occup Med. 1993;35:574-576.

Sina D, Schuhmann R, Abraham R, Taubert HD, Dericks-Tan JS. Increased serum FSH levels correlated with low and high sperm counts in male infertile patients. Andrologia. 1975;7:31-37.

Subhan F, Tahir F, Ahmad R, Khan ZD. Oligospermia and its relation with hormonal profile. J Pak Med Assoc. 1995;45:246-247.

Uhler ML, Zinaman MJ, Brown CC, Clegg ED. Relationship between sperm characteristics and hormonal parameters in normal couples. Fertil Steril. 2003;79(suppl 3):1535-1542.

Vander A, Sherman J, Luciano D. Human Physiology: The Mechanisms of Body Function. 7th ed. Boston, Mass: McGraw-Hill; 1998.

Veldhuis JD, King JC, Urban RJ, Rogol AD, Evans WS, Kolp LA, Johnson ML. Operating characteristics of the male hypothalamopituitary-gonadal axis: pulsatile release of testosterone and folliclestimulating hormone and their temporal coupling with luteinizing hormone. J Clin Endocrinol Metab. 1987;65:929-941.

Vermeulen A, Verdonck G. Representativeness of a single point plasma testosterone level for the long term hormonal milieu in men. J Clin Endocrinol Metab. 1992;74:939-942.

World Health Organization. WHO Laboratory Manual for the Examination of Human Semen and Sperm-Cervical Mucus Interactions. New York: Cambridge University Press; 1999. 